\title{
Structure and stability of bosonic clouds: alkali atoms with negative scattering length
}

\author{
A. Parola ${ }^{1,2}$, L. Salasnich ${ }^{1,3}$, L. Reatto ${ }^{1,3}$ \\ ${ }^{1}$ Istituto Nazionale per la Fisica della Materia, Unità di Milano, Via Celoria 16, Milano, Italy \\ ${ }^{2}$ Istituto di Scienze Fisiche, Universitá di Milano, Via Lucini 3 Como, Italy \\ 3 Dipartimento di Fisica, Universitá di Milano, Via Celoria 16, 20133, Milano, Italy
}

\begin{abstract}
We investigate the form and stability of a cloud of atoms confined in a harmonic trap when the scattering length is negative. We find that, besides the known low density metastable solution, a new branch of Bose condensate appears at higher density when non locality effects in the attractive part are taken into account. The transition between the two classes of solutions as a function of the number $N$ of atoms can be either sharp or smooth according to the strength and range of the attractive interaction. Use of tight traps is favorable for investigating the evolution of the system as the strength of the effective interaction increases with $N$.
\end{abstract}

03.75.Fi, 05.30.Jp, 32.80.Pj

Typeset using REVTEX 
Few alkali isotopes are characterized by a negative scattering length $a_{T}$ at low energy in the triplet channel [1]. This is believed to have important consequences on the existence and the structure of the Bose Einstein condensed (BEC) state for trapped clouds of atoms when the isotope follows Bose statistics. The most celebrated case is ${ }^{7} \mathrm{Li}$ for which there is experimental evidence in favor of a BEC state if the number of atoms does not exceed a threshold [2]. This behavior was in fact predicted theoretically. When the interaction between atoms is represented by an effective local potential $v_{\text {eff }}(\mathbf{r})=\left(4 \pi \hbar^{2} / m\right) a_{T} \delta^{3}(\mathbf{r})$, a uniform gas phase is unstable towards the formation of collapsed state of high density. The situation changes in presence of an external trap [3]. Within the Gross-Pitaevskii (GP) equation for the condensate wavefunction [4], the kinetic energy due to the confinement in the trap opposes the collapse as long as the number $N$ of atoms does not exceed a critical number $N_{c}$ which depends on the parameters of the system (isotope mass $m$, scattering length $a_{T}$, shape of the trap, etc.) [5]. Even for $N<N_{c}$, we must recall that a dilute cloud represents a metastable state which can be experimentally observed only if its lifetime is long enough.

The possibility of having positive as well as negative scattering lengths in different atomic systems arises because the potential between a pair of atoms generally supports bound states leading to the formation of a dimer with several vibrational levels. Under this condition, $\left|a_{T}\right|$ can be very large but at the same time the energy dependent scattering cross section $\sigma_{T}(E)$ starts to deviate from its zero energy limit $\sigma_{T}(0)=4 \pi a_{T}^{2}$ already at an energy that can be extremely small [6]. The calculations by Côté et al. [7] for ${ }^{7} \mathrm{Li}$ show that $\sigma_{T}(E)$ is reduced to $50 \%$ of $\sigma_{T}(0)$ at energies of the order of $310^{-9}$ a.u. . This value is small enough that $s$-wave scattering is dominant over higher angular momentum states meaning that the scattering process between two atoms can still be represented by an effective two body interaction $v_{\text {eff }}$. However, in general we cannot neglect the momentum dependence of $v_{\text {eff }}(k)$ for the colliding atoms. This amounts to say that the effective interaction is non local: In this note we investigate the effects of non-locality on the stability of the cloud.

It is rather easy to convince that non-locality in the interatomic effective potential can 
modify the stability condition of the Bose condensate. The GP functional $\mathcal{E}[\Psi]$ with non local interaction $v_{\text {eff }}(\mathbf{r})$ reads:

$$
\begin{aligned}
\mathcal{E}[\Psi]= & \int d^{3} \mathbf{r} \frac{\hbar^{2}}{2 m}|\nabla \Psi|^{2}+\int d^{3} \mathbf{r} U_{\text {ext }}(\mathbf{r})|\Psi(\mathbf{r})|^{2}+ \\
& \frac{1}{2} \int d^{3} \mathbf{r} \int d^{3} \mathbf{r}^{\prime}\left|\Psi\left(\mathbf{r}^{\prime}\right)\right|^{2} v_{\text {eff }}\left(\mathbf{r}-\mathbf{r}^{\prime}\right)|\Psi(\mathbf{r})|^{2}
\end{aligned}
$$

where $\Psi(\mathbf{r})$ is the wavefunction of the condensate and $U_{\text {ext }}(\mathbf{r})$ is the potential of the trap. In this paper we consider a symmetric harmonic trap $U_{\text {ext }}(\mathbf{r})=\frac{1}{2} m \omega_{0}^{2} r^{2}$. In the local limit, one recovers the standard form of the GP functional. The ground state wavefunction of a cloud of $N$ atoms is determined by minimizing $\mathcal{E}[\Psi]$ with the constraint that

$$
\int d^{3} \mathbf{r}|\Psi(\mathbf{r})|^{2}=N
$$

In the ground state $\Psi(\mathbf{r})$ is positive definite and spherically symmetric.

As a first step, we discuss an approximate, variational approach to this problem which already shows the main features of the exact solution. As a trial wavefunction we choose a Gaussian form:

$$
\Psi(\mathbf{r})=N^{\frac{1}{2}}\left(\frac{\lambda^{2}}{\pi a_{H}^{2}}\right)^{\frac{3}{4}} \exp \left(-\frac{\lambda^{2} r^{2}}{2 a_{H}^{2}}\right)
$$

with a single variational parameter $\lambda$ which defines the size of the cloud in units of the harmonic oscillator length $a_{H}=\left[\hbar /\left(m \omega_{0}\right)\right]^{1 / 2}$. By substituting $\mathbf{r}^{\prime}=\mathbf{r}+\mathbf{s}$ in Eq. (11) and developing $\Psi(\mathbf{r}+\mathbf{s})$ in powers of $\mathbf{s}$, we perform a gradient expansion of the energy functional $\mathcal{E}[\Psi]$. To lowest order in the gradient of the wavefunction, the energy reads:

$$
\mathcal{E}(\lambda)=\frac{\hbar \omega_{0}}{2} N\left(\frac{3}{2} \lambda^{2}+\frac{3}{2} \lambda^{-2}+\gamma \lambda^{3}+\gamma \tau \lambda^{5}\right)
$$

where the two parameters $\gamma$ and $\tau$ depend on the choice of the effective interaction providing a dimensionless estimate of the strength $\gamma$ and range $\tau$ of $v_{\text {eff }}(r)$ :

$$
\begin{aligned}
\gamma & =\sqrt{2 / \pi} N a_{T} a_{H}^{-1} \\
\tau & =-\left[3 \int d r r^{4} v_{\mathrm{eff}}(r)\right]\left[2 a_{H}^{2} \int d r r^{2} v_{\mathrm{eff}}(r)\right]^{-1}
\end{aligned}
$$


According to these definitions, $\tau$ is basically minus the square of the range of the effective interaction and is therefore a negative quantity. For a local interaction $\tau=0$ and we recover Baym and Pethick result [5]. In this limit and for negative scattering length, $\gamma<0$ and $\mathcal{E}(\lambda)$ goes to $-\infty$ for $\lambda \rightarrow \infty$ leading to a collapsed ground state. However, $\mathcal{E}(\lambda)$ has a local minimum for a finite value of $\lambda$ when $|\gamma|<4 / 5^{5 / 4}$, i.e. when $N<N_{c} \sim 0.67 a_{H}\left|a_{T}\right|^{-1}$. This represents the metastable state mentioned above.

It is clear from Eq. (蛋) that non-locality changes the stability condition because, for negative scattering length, the product $\gamma \tau$ is positive. Depending on the value of the parameters, $\mathcal{E}(\lambda)$ can have one or two minima and the collapse is prevented in any case. However, this result is only suggestive because the gradient expansion breaks down as soon as non-locality becomes important and a more appropriate formalism is needed. We have then studied the variational equation obtained with the same choice of a Gaussian wavefunction (3) but without invoking gradient expansion. This problem requires the explicit definition of the effective interaction. We assume that the attractive potential has a finite range $r_{e}$ and in addition we allow for the presence of a repulsive contribution which is modeled as a local positive term defined by a "high energy" scattering length $a_{R}>0$. With this choice, $v_{\text {eff }}(k)$ changes sign from negative at small $k$ (i.e. low energy), to positive at larger $k$ thereby mimicking the microscopic computations of the $\ell=0$ phase shift $\delta_{k}$ which show a change in sign from positive to negative when $k$ increases [6]. At still higher momenta, scattering in other channels with $\ell \neq 0$ becomes important but we assume that the range of density is such that this regime is never reached.

In the model we study, the effective interaction is then written in the following form:

$$
v_{\text {eff }}(k)=\frac{4 \pi \hbar^{2}}{m}\left[a_{R}+\left(a_{T}-a_{R}\right) f\left(k r_{e}\right)\right]
$$

We have considered two choices for the shape function $f(x)$ : A Lorenzian $f(x)=\left(1+x^{2}\right)^{-1}$ and a Gaussian $f(x)=\exp \left(-x^{2}\right)$. The results do not depend on the specific choice of $f(x)$ and so we will discuss only the Lorenzian case. We use interaction parameters appropriate for ${ }^{7} \mathrm{Li}: a_{T}=-27 a_{B}$ [8], $r_{e}=10^{3} a_{B}$ [7] and $a_{R}=6.6 a_{B}$ [9] (where $a_{B}$ is the Bohr radius). 
The energy functional for the Lorenzian potential can be analytically expressed in terms of elementary functions and reads:

$$
\begin{aligned}
\mathcal{E}(\lambda)= & \frac{\hbar \omega_{0}}{2} N\left\{\frac{3}{2} \lambda^{2}+\frac{3}{2} \lambda^{-2}+\gamma_{R} \lambda^{3}-\tau_{1} \lambda+\right. \\
& \left.+\tau_{2} \operatorname{erfc}\left(\chi \lambda^{-1}\right) \exp \left(\chi^{2} \lambda^{-2}\right)\right\}
\end{aligned}
$$

where

$$
\begin{aligned}
\gamma_{R} & =N \sqrt{\frac{2}{\pi}} \frac{a_{R}}{a_{H}} & \tau_{1} & =N \sqrt{\frac{2}{\pi}} a_{H} \frac{a_{T}-a_{R}}{r_{e}^{2}} \\
\chi & =\frac{a_{H}}{\sqrt{2} r_{e}} & \tau_{2} & =N a_{H}^{2} \frac{a_{T}-a_{R}}{r_{e}^{3}}
\end{aligned}
$$

and $\operatorname{erfc}(x)=1-\operatorname{erf}(x)$ is the complementary error function. The extrema of $\mathcal{E}(\lambda)$ are obtained as solutions of an algebraic equation. This equation has either one or three positive roots depending on the parameters and on number $N$ of atoms in the cluster. When three solutions are present, the intermediate one represents an unstable state (i.e. a local maximum of the energy) while the other two respectively describe a low density metastable solution and a minimum which represents the stable solution within GP approximation.

The variational results for three typical trap sizes are shown in Fig. 1 where the average radius and the density at the center of the cloud are plotted as a function of $N$. In the same figure, the variational data are also compared with the exact solution of the GP equation, obtained by numerical integration of the corresponding self-consistent Schrödinger equation. In fact, the exact minimization of the GP functional (1) gives rise to a non linear eigenvalue problem for the ground state wavefunction. The ground state is spherically symmetric and can be written as $\Psi(\mathbf{r})=\phi(r) / r$ where $\phi(r)$ satisfies:

$$
-\frac{\hbar^{2}}{2 m} \frac{\mathrm{d}^{2}}{\mathrm{~d} r^{2}} \phi(r)+\frac{m \omega_{0}^{2}}{2} r^{2} \phi(r)+W(r) \phi(r)=\epsilon \phi(r)
$$

Here $\epsilon$ is the chemical potential and

$$
W(r)=\int d^{3} \mathbf{r}^{\prime}\left|\phi\left(r^{\prime}\right)\right|^{2} v_{\text {eff }}\left(\mathbf{r}-\mathbf{r}^{\prime}\right)
$$

is the self-consistent interaction. For effective potentials written as sums of Yukawa functions, this integro-differential equation can be reduced to a boundary value problem for a 
set of ordinary differential equations. In particular, in the case of interest, the form (6) gives rise to three ordinary differential equations which have been integrated by standard Runge-Kutta method.

Fig. 1 shows the good performance of the variational approach which is always very close to the exact solution. For large $N$ the size of the cloud is remarkably independent of the trap size demonstrating that the atoms are in a self-bound configuration. The size of the cloud is governed by the effective range: For large values of $r_{e}\left(r_{e} \gg\left|a_{T}\right|\right)$ and $N$ not too large, the reduced density $\rho\left|a_{T}\right|^{3}$ is small. This indicates that the self bound state that we find represents a novel regime of the cloud, intermediate between the very low density state already predicted within the approximation of local interaction and the collapsed high density state which depends on the detailed shape of the true interatomic potential [10].

The asymptotic large $N$ behavior of the exact solution can be obtained analytically from our equation: The radius of the cloud reaches a finite limit which coincides with the result of the Thomas-Fermi approximation to the GP equation [11]. On the other hand, the low density branch is virtually indistinguishable from the exact solution of a local attractive potential, also shown in figure. The effects of non-locality become important just when the radius of the cloud rapidly drops. This "transition" is discontinuous for large traps, where the reentrant behavior of the curve shows the presence of an unstable branch. By reducing the trap size, however, this discontinuity is strongly reduced and, below $0.3 \mu \mathrm{m}$, the unstable branch disappears and there is a smooth evolution from a very dilute cloud to a less dilute state with an increasing density as $N$ grows.

The predictions of the standard treatment of clouds of alkali atoms in terms of a local pseudopotential have to be modified in two respects when the scattering length is negative. In the case of shallow traps, the stability threshold $N_{c}$ is only an upper bound. There is a lower threshold $N_{c l}$ for the higher density state and in the intermediate region $N_{c l}<N<N_{c}$ the low density branch is metastable towards the higher density solution. For instance, in a $3 \mu \mathrm{m}$ trap $N_{c}=1300$ and $N_{c l}=160$. Which state is reached experimentally depends on how the cloud is formed. In the case of a tight trap a threshold does not exist altogether. This 
feature allows to explore experimentally how the state of the system evolves as the interaction strength increases and depletion effects start to set up even at the lowest temperature. It should be noticed that the presence of a repulsive short range interaction in our model potential does not play a crucial role. The higher density branch as well as the existence of two regimes depending on the size of the trap are present even if we put $a_{R}=0$. The only difference is that, in this case, the radius of the cloud slowly decreases as $N \rightarrow \infty$ and does not reach a finite limit as in Fig. 1. In any case, it should be kept in mind that in the large $N$ limit our results are only qualitative because the GP equation itself breaks down and interaction effects are expected to produce a depletion of the condensate when $\rho\left|a_{T}\right|^{3}$ is not very small. However, we believe that the trend shown by this equation is significant even if quantitative results must wait for a more accurate determination of the interatomic potential and a better treatment of of the many body problem.

Another interesting problem which can be addressed by this formalism is the shape of the condensate wavefunction. This information may be useful for the quantitative determination of the number of atoms in the condensate from experimental data. The GP ground state wavefunction, normalized to unity at the origin, is shown for different numbers of atoms in the cloud in Fig. 2a. We see that some deviation from a Gaussian form develops as density grows. In particular, a sharp peak at the cloud center grows at large $N$. Along the very low density branch we verified that the wavefunction is not affected by the non locality of the interaction and is very close to the numerical result in the local approximation. However, significant deviations appear as soon as we approach the edge of the low density branch.

As a next step we can investigate the dependence of the shape of the equilibrium curve when the range of the attractive interaction is varied. In fact, we expect that the larger is this lengthscale, the stronger is the effect of non locality in the stability diagram of the cloud. More importantly, the value of the effective range $r_{e}$ is rather uncertain even in Lithium and it is useful to test the sensitivity of our results to variations of the shape of the effective interaction. In Fig. 2b we show the equilibrium curves of a cloud of Lithium atoms in a harmonic trap of $a_{H}=1 \mu \mathrm{m}$ when the effective range $r_{e}$ is artificially halved or doubled 
with respect to the reference value $r_{e}=10^{3} a_{B}$ previously adopted. The results clearly show a smoothing of the transition between the two branches induced by the enhancement of non locality. By increasing the effective range, the maximum number of atoms in the low density branch $N_{c}$ slightly increases and the two branches of solutions tend to merge into a unique phase smoothly connecting the low and the higher density regimes. A quantitative analysis of the variational solution shows that the occurrence of the single branch regime is mainly determined by the ratio between the trap radius and the effective range, independently of the scattering length: $a_{H}<5 r_{e}$. Conversely, the number of atoms in the cloud at the inflection point can be estimated as $N_{c} \sim 4.5 r_{e}\left|a_{T}\right|^{-1}$, independently of the trap radius. These results show that the effective range plays a crucial role in determining the stability of the bosonic cloud at intermediate density. The previous estimates will become particularly valuable when an accurate determination of the interaction parameters will become available also for other alkali isotopes with negative scattering length. Interestingly, new techniques are being devised in order to modify the effective interaction in these systems [12].

In conclusion, the analysis of the Bose-Einstein condensed ground state of Lithium atoms in a harmonic trap has shown that atomic clouds may exist in a novel state, intermediate in density between the very dilute regime which has been studied up to now and the collapsed high density state which is determined by the short range behavior of the true interatomic potential. Also this intermediate state is self-bound because the size of the cloud is essentially independent of the size of the trap. The importance of the study of this intermediate regime arises from the possibility of tuning the strength of the interaction by changing $N$ : In this way it will be possible to study how the depletion of the condensate affects the structure and the physical properties of the state. To this end, it is important to confine the system in sufficiently small traps in order to avoid the discontinuous jump between the two phases. Interestingly, such micro-traps are under development [13]. Non locality in the effective interaction is a crucial ingredient which prevents collapse when the scattering length is negative. Long range potentials favor the formation of big clouds which still maintain a rather low density. Therefore, it is quite interesting to select elements and isotopes characterized 
by negative scattering length together with long range effective interactions. We stress that our results apply to the dilute regimes where GP equation correctly represents the behavior of the ground state. At higher density, collisions between atoms deplete the condensate. In addition three body scattering starts to play a role and spin-flip processes lead to rapid destruction of the cloud.

We acknowledge financial support from the "BEC" advanced research project of INFM. 


\section{REFERENCES}

[1] A. J. Moerdijk et al Phys. Rev. Lett. 72, 40 (1994); B.J. Verhaaret al Phys. Rev. A 48, R3429 (1993).

[2] C.C. Bradley et al Phys. Rev. Lett. 78, 985 (1997).

[3] P.A. Ruprecht et al Phys. Rev. A 51, 4704 (1995).

[4] E.P. Gross, Nuov. Cim. 20, 454 (1961); L.P. Pitaevskii, Sov. Phys. JETP 13, 451 (1961).

[5] G. Baym and C.J. Pethick, Phys. Rev. Lett. 76, 6, (1996); A.L. Fetter Phys. Rev. A 53, 4245 (1996); F. Dalfovo and S. Stringari, Phys. Rev. A 53, 2477 (1996).

[6] G.F. Gribakin and V.V. Flambaum, Phys. Rev. A 48, 546 (1993).

[7] R. Côté, A. Dalgarno and M.J. Jamieson Phys. Rev. A 50, 399 (1994).

[8] E.R.I. Abraham et al Phys. Rev. Lett. 74, 1315 (1995).

[9] This choice for $a_{R}$ coincides with the zero of the microscopic interatomic potential of Ref. [7].

[10] H.T.C. Stoof Phys. Rev. A 49, 3824 (1994).

[11] M. Lewenstein and L. You, Phys. Rev. A 53, 909 (1996).

[12] P.O. Fedichev et al Phys. Rev. Lett. 77, 2913 (1996).

[13] T. Haensch, private communication (1997). 


\section{Figure captions}

Fig. 1 Average radius of the cloud $R$ (panels a,c,e) and central density (panels b,d,f) as a function of number of atoms $N$ for the trap sizes $a_{H}=3 \mu \mathrm{m}, 1 \mu \mathrm{m}$ and $0.3 \mu \mathrm{m}$ respectively. Full dots represent the numerical solution of the GP equation with non local Yukawa potential. Open dots correspond to the local approximation. Solid line is the variational result and the dashed line is the asymptotic limiting radius for $N \rightarrow \infty$. Parameters have been chosen as to mimic ${ }^{7} \mathrm{Li}$.

Fig. 2 Panel (a): Condensate wavefunction $\Psi(r)$ for a trap size $a_{H}=0.3 \mu \mathrm{m}$ and non local interaction. The non interacting result (Gaussian wavefunction) would be a straight line in this plot. Dashed line: $N=68$ (low density). Dotted line: $N=159$ (near the maximum of the low density branch). Solid line: $N=166$ (higher density branch). Panel (b): Equilibrium curves for parameters appropriate for Lithium atoms in a trap of $a_{H}=1 \mu \mathrm{m}$

and three choices of the effective range of the Lorenzian attractive potential. Variational results. Dotted line: $r_{e}=500 a_{B}$. Solid line $r_{e}=1000 a_{B}$. Dashed line $r_{e}=2000 a_{B}$. 

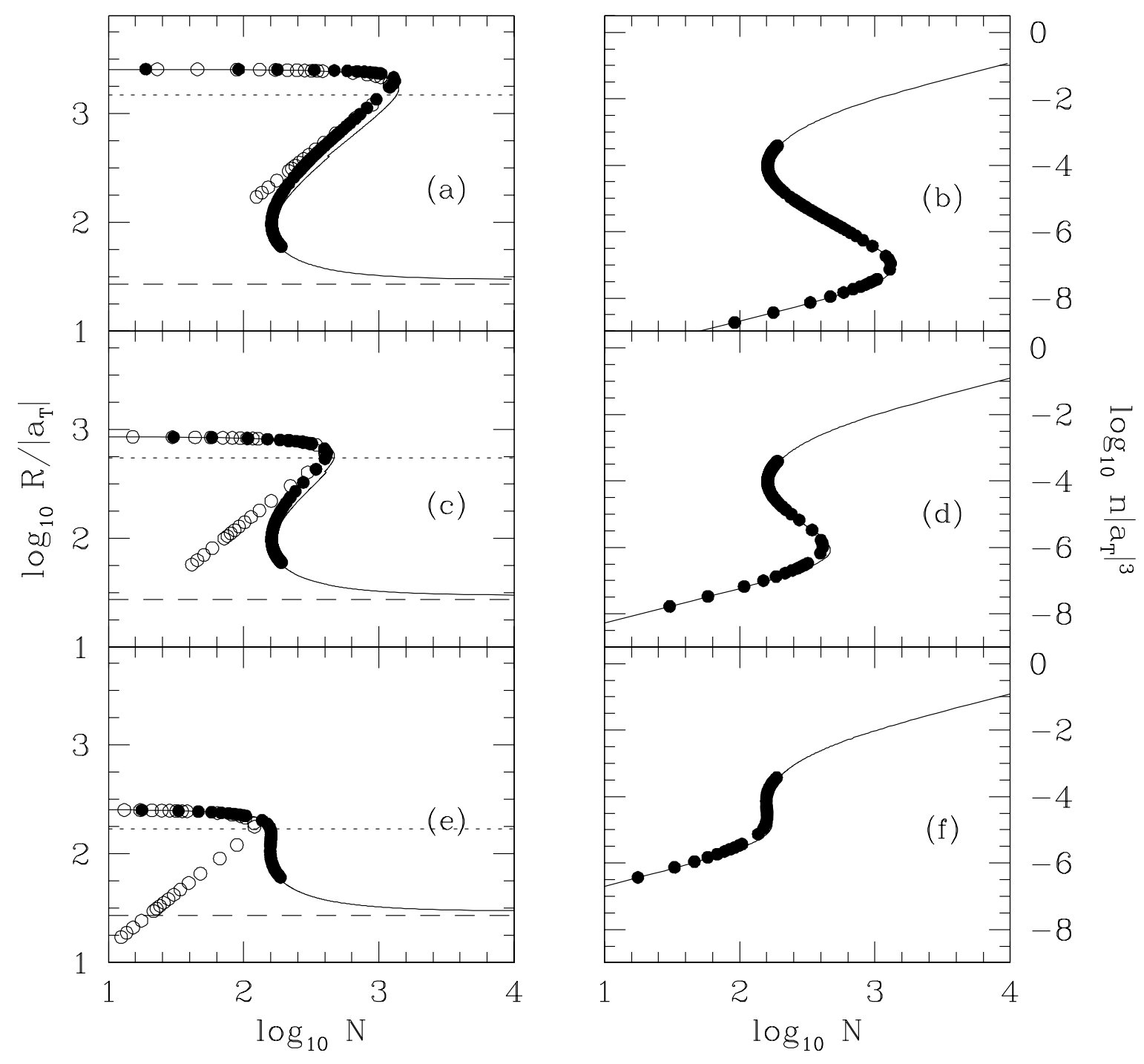

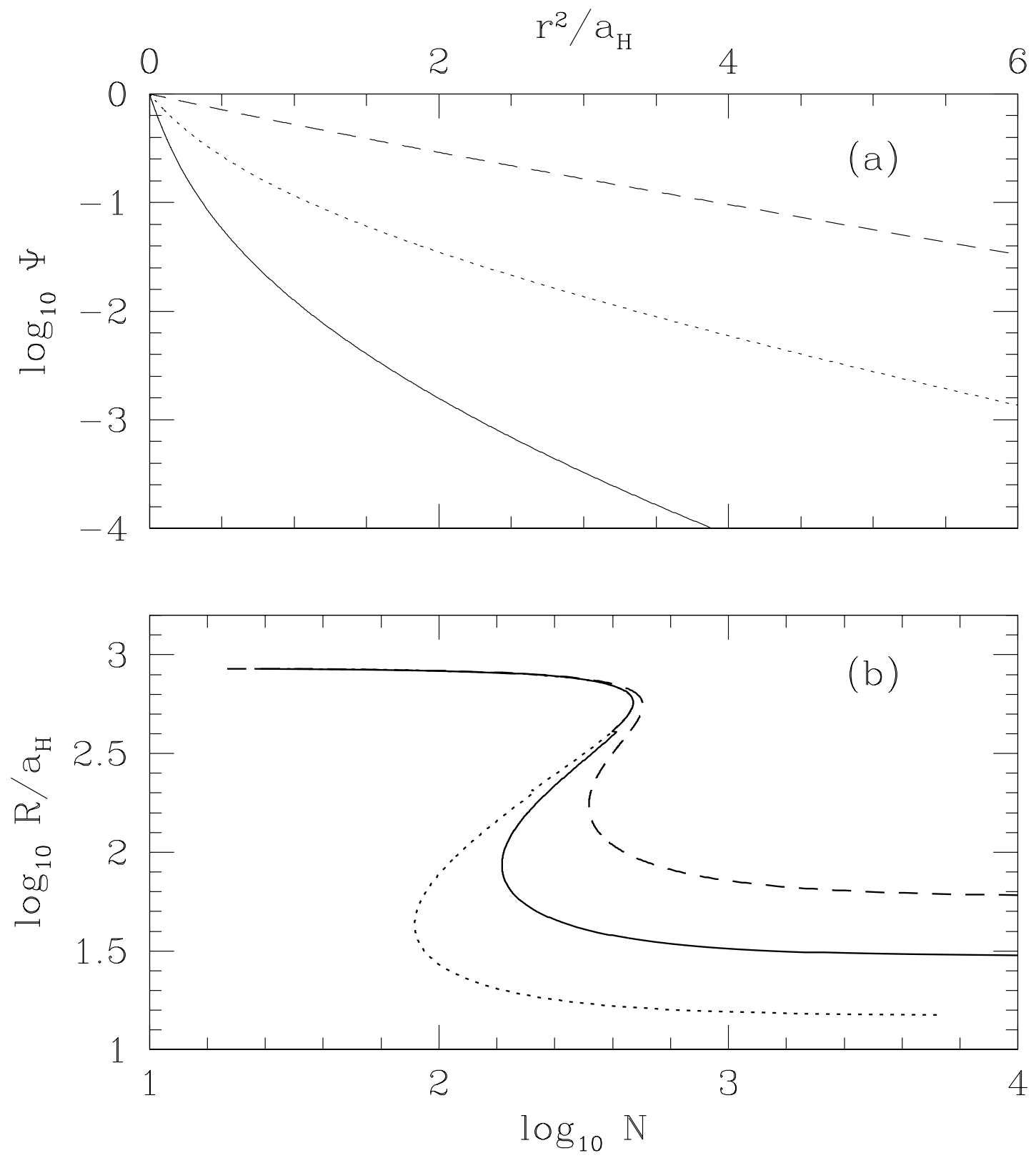